\title{
The Cartilage Rhinoplasty with Goldman Technique for Correcting the Broad Nasal Tip
}

\author{
Ming He, Teng Zhao* \\ Zhoukou Vocational and Technical College, Zhoukou, Henan, China \\ *Corresponding Author.
}

\begin{abstract}
:
In this paper, A retrospective review was performed for all the patients who underwent surgery with the Goldman technique by the senior author from 2004 to 2009 for correction of the broad nasal tip. The clinical and pathologic findings of these patients were reviewed, and an independent observer evaluated the pre- and postoperative photos of the patients using five parameters: projection, rotation, symmetry, shape, and distance of the tip-defining points. The evaluation was performed using a scale of -1 to +1 for each of the five parameters. Of the 205 patients who underwent the technique, 115 (56\%) were patients with broad tips, and 90 (44\%) were patients with broad (boxy) tips. A total of 189 cases (92.2\%) involved primary rhinoplasties, and 16 cases $(7.8 \%)$ involved revision. During this period, complications were observed in five patients $(2.4 \%)$, who underwent revision rhinoplasty with a successful result. The average score for the five parameters already mentioned for primary rhinoplasties according to the scale of -5 to +5 showed a significant postoperative improvement (score, +4.3 ). It implied that the cartilage rhinoplasty with Goldman technique is safe when performed by experienced surgeons and according to specific indications. This conclusion is indicated by the low rate of complications in the large series of patients in this study.
\end{abstract}

Keywords: Cartilage rhinoplasty; Goldman technique; Correcting; Broad nasal tip; Dome-splitting

\section{INTRODUCTION}

The aesthetic importance of the nose should not be underestimated it is a key determinant of the first impressions people have about each other [1]. Therefore, many people wish to alter their typically flat or broad noses though surgery. Although many plastic surgeons have endeavored to create a beautiful and ideal nose shape through surgery, it is difficult to give the ideal nose, which is small and flat with a bulbous nasal tip, a natural-looking, nicely projecting nasal tip 
Article History: Received: 28 October 2021 Revised: 05 December 2021 Accepted: 10 January 2022 Publication: 28 February 2022

with a long and high dorsum. It is obvious that the rhinoplasty is the most challenging objective in rhinoplasty because it plays a very significant role in the achievement of a functional, aesthetically pleasing, and natural result [2]. Many techniques have been proposed in the past for the correction of tip deformities. The surgeon must have the technical skills required and the experience to take advantage of them to achieve the best aesthetic and functional result [3].

During the past 20 years, a more conservative spirit has been observed concerning surgical maneuvers in the nasal tip because it is believed that the more aggressive techniques, although effective in manipulating the tip, do not have a predictable postoperative result [4-5]. Modern rhinoplasty surgeons appear hesitant to use methods that distract from the normal tip architecture and prefer to perform the multiple-suture techniques that have been described [6], as well as various grafts, for the correction of tip abnormalities [7].

Suturing techniques for the nasal tip (suture modification of the lower lateral cartilages) are a reliable method for correcting the bulbous nasal tip and controlling tip projection or rotation [89]. However, as Toriumi and Tardy have mentioned, if cartilage suturing techniques prove to be inadequate, then more aggressive techniques such as dome division can be used.

In 1957, Goldman first described division of the lower lateral cartilages immediately lateral to the dome to create bilateral, symmetric medial and lateral strips. The medial strips were advanced anteriorly and sewn together to create a strong medial unit, increasing projection and strengthening medial tip support [10]. The now discontinuous lateral strips were allowed to fall away posteriorly. This method has been used during the past 50 years to correct tip abnormalities such as broad (bulbous) nasal tip and tip under-projection or over-projection. However, it has been associated with problems such as notching, pinching of the tip, and tip asymmetries [11].

The goal of this report is to prove, through the review of a large series of patients who had surgery with the Goldman technique by the senior author (A.S.), that this method is safe when performed by experienced surgeons and with specific indications.

\section{RESEARCH METHODS}

We performed a retrospective review of the patients who underwent rhinoplasty by the senior author (A.S.) from 2004 to 2009. Our database included the patients' pathologic clinical findings, their pre- and postoperative photos, and the data from their postoperative follow-up evaluation. In this study, we included 205 patients for whom the Goldman technique was performed to correct the over-projected or the bulbous tip. The follow-up period ranged from 12 to 60 months.

The pre- and postoperative photos were evaluated by an independent observer using five parameters: (1) satisfactory tip projection; (2) tip shape (triangular vs. trapezoid); (3) tip symmetry; (4) appropriate tip rotation; and (5) presence and distance of tip-defining points. 
Article History: Received: 28 October 2021 Revised: 05 December 2021 Accepted: 10 January 2022 Publication: 28 February 2022

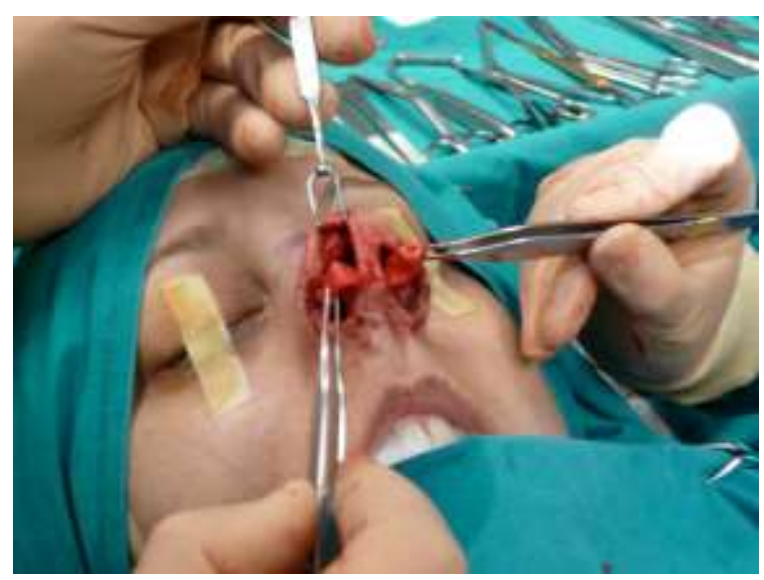

Fig. 1 Delivery of the domes

Tip projection was evaluated using the Goode ratio and tip rotation with measurement of the nasolabial angle (95-100 degrees for females and 90-95 degrees for males). The distance between the tip-defining points was considered improved when it had decreased by at least $2 \mathrm{~mm}$ postoperatively. For each patient, a score of $-1,0$, or +1 was given for each of the five parameters depending on whether they had deteriorated, stayed the same, or improved respectively with the Goldman technique. The maximum score for each patient was +5 , which showed postoperative improvement in all five categories, and the minimum score was -5 .

All patients underwent surgery using the endonasal approach. With this approach, after the marginal and transfixion incision, the two domes are delivered (Fig. 1), and the alar cartilages are reduced cephalically in a conservative manner to narrow the tip without compromising the function of the external valve. The width of the lateral crura after its cephalic reduction should always be at least $5-8 \mathrm{~mm}$.

Next, the surgeon marks the apex of the domes of the lower lateral cartilages and performs the vertical division of the alar cartilages, in all cases $1.5 \mathrm{~mm}$ laterally to the apex of the domes (Figs. 2, 3). The dissection includes the underlying skin. 
Article History: Received: 28 October 2021 Revised: 05 December 2021 Accepted: 10 January 2022 Publication: 28 February 2022
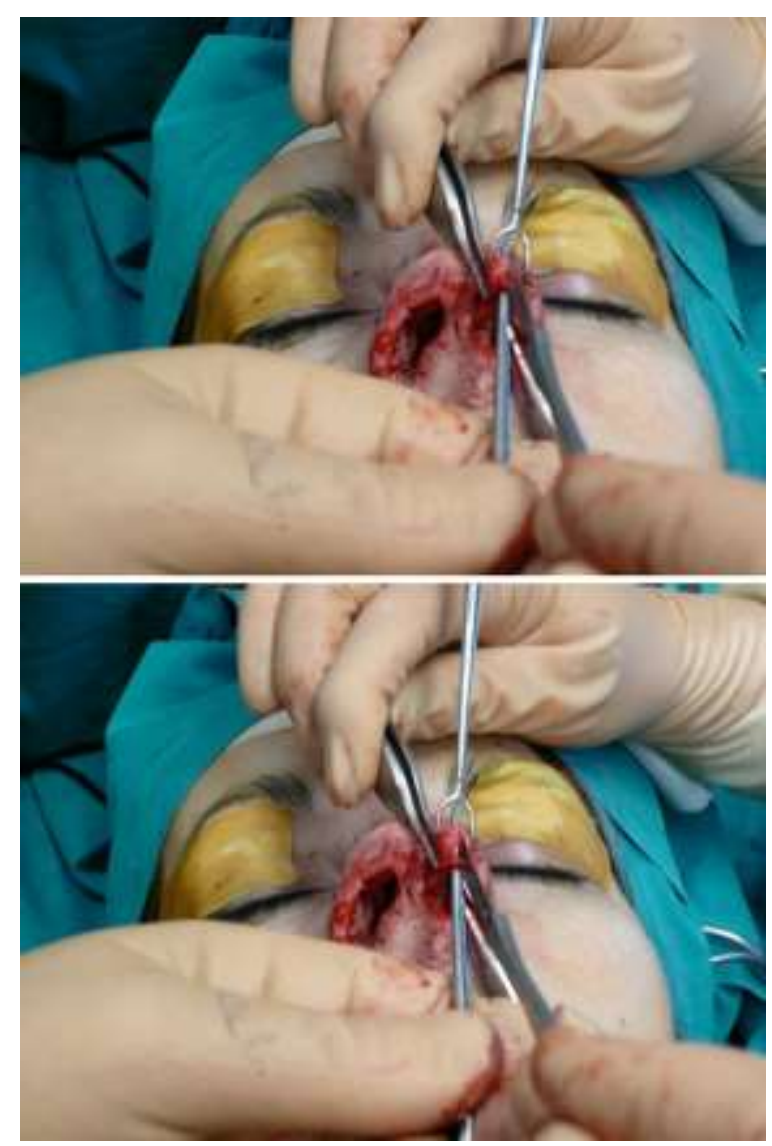

Figs. 2-3 Vertical division of the alar cartilages

In case the underlying defect is an over-projected tip, the height of the central cartilagecutaneous flap (consisting of the medial crura with the overlying skin) is reduced (Fig. 4). With this maneuver, the nasal tip is over-projected. In the cross section, the lateral crus usually is long and overlaps the new dome. This overlap should be trimmed so that the lateral crus lines up in continuance with the edge of the new dome cartilage (Fig. 5).

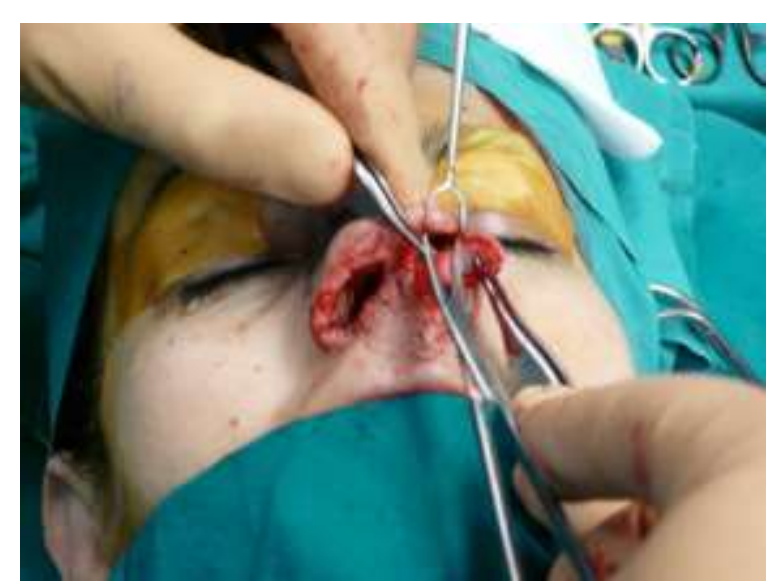


Article History: Received: 28 October 2021 Revised: 05 December 2021 Accepted: 10 January 2022 Publication: 28 February 2022

Fig. 4 If the underlying defect is an over-projected tip, the height of the central cartilagecutaneous flap is reduced

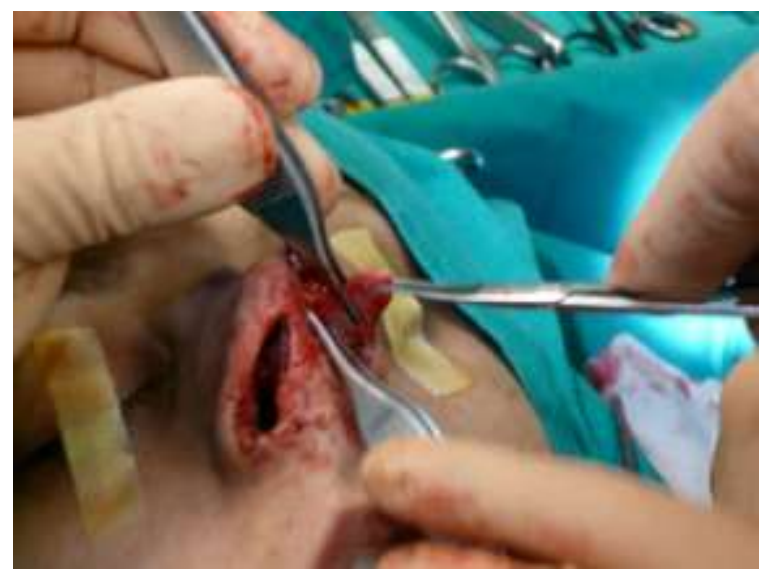

Fig. 5 The lateral crus after the transection usually is long and overlaps the new dome. This overlap should be trimmed so the lateral crus will line up in continuance with the edge of the new dome cartilage

The two medial crura then are sutured together with an absorbable 5-0 PDS suture. This suture is placed $0.2-0.3 \mathrm{~cm}$ below the apex of the medial crura to maintain the normal curvature of the domes and to avoid the formation of an unnatural thin or pinched tip. When the tip support is weak, usually in noses with a short or deviated septum or with medial crura that are short or curved and footplates that do not reach down to the nasal spine, a columellar strut is placed between the medial crura. The lateral flaps are sutured in place with the overlying skin using Vicryl 6-0 absorbable sutures both to ensure their approximation to the medial flaps and to avoid their displacement or malposition (Figs. 6, 7, 8, 9). 
Article History: Received: 28 October 2021 Revised: 05 December 2021 Accepted: 10 January 2022 Publication: 28 February 2022
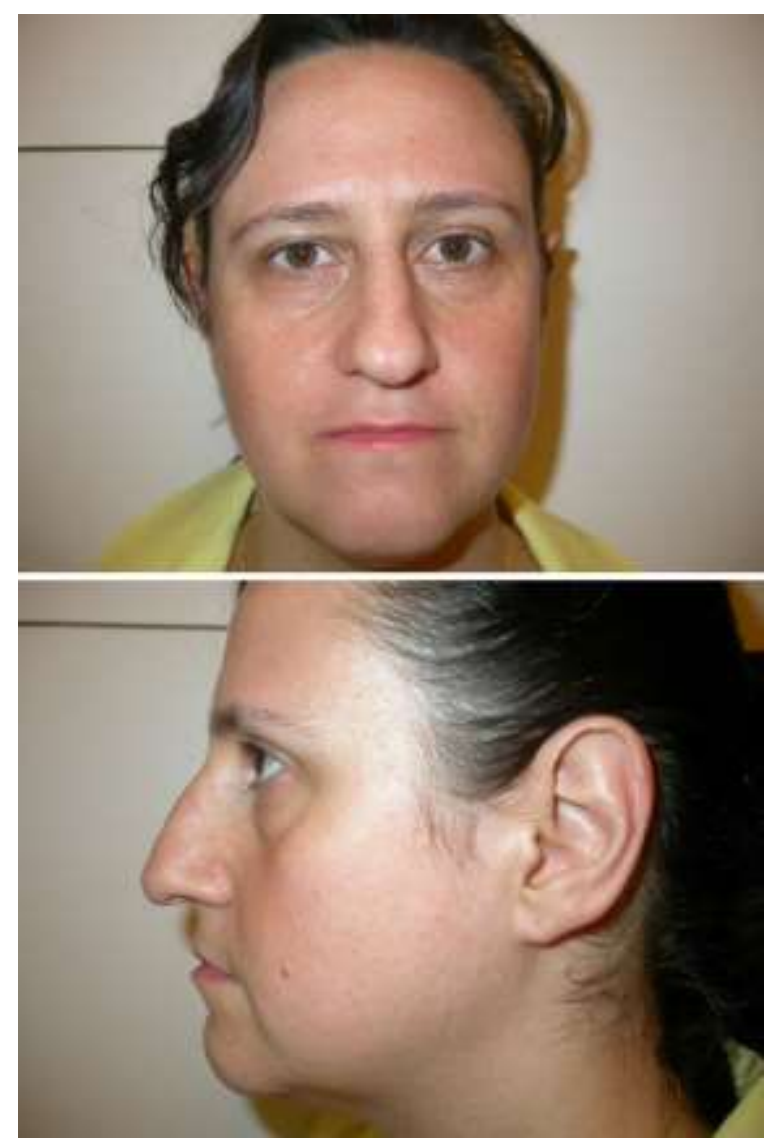

Figs. 6-7 Patient with broad bulbous tip

\section{RESULTS}

Among the 2,708 rhinoplasty cases reviewed in our database, the Goldman technique was performed for 205 patients $(7.5 \%)$ to correct the over-projected or bulbous tip. The patients' individual features are shown in Table 1 . A total of 115 cases $(56 \%)$ involved noses with overprojected tips, whereas 90 patients $(44 \%)$ had a bulbous tip.

Of the 205 cases, $189(92.2 \%)$ were primary and $16(7.8 \%)$ were revision rhinoplasties. The 205 patients comprised 160 women (78\%) and 45 men (22\%) with a mean age of 33 years (range, 20-55 years). The follow-up period ranged from 1 to 5 years (mean, 3 years). During this period, complications were observed in five patients (2.4\%) (Table 2). Alar collapse was considered a consequence of an aggressive cephalic reduction of the lateral crura. Tip under-projection resulted from the larger reduction in the height of the central flap. Finally, a pinched tip was observed in a woman with very thin skin. In this case, the surgeon had sutured the two medial crura together closer to their apex than the usual 2- to 3-mm distance, a fact that also contributed to the pinching of the tip. All five patients underwent reoperation with successful results. 
Article History: Received: 28 October 2021 Revised: 05 December 2021 Accepted: 10 January 2022 Publication: 28 February 2022
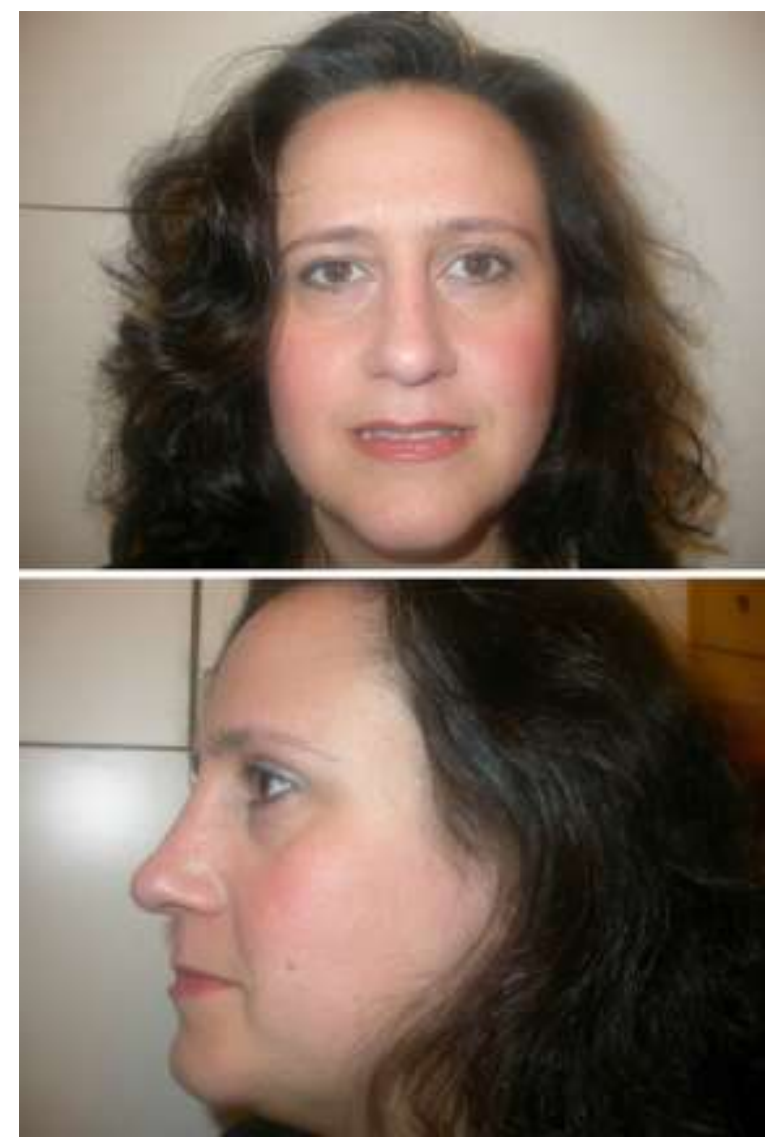

Figs. 8-9 The patient in Figs. 6 and 7 viewed 1 year postoperatively. The Goldman technique was used

The evaluation of the pre- and postoperative photos by the independent observer using the aforementioned five parameters is presented in Table 3 for the primary cases and in Table 4 for the revision cases. The mean evaluation score of these five parameters for primary rhinoplasties according to the scale of -5 to +5 shows a significant postoperative improvement (score, +4.3 ). For revision rhinoplasties, the results are presented in Table 4, which shows a greater improvement (score, +4.5).

TABLE I. Characteristics of patients treated with the Goldman technique

\begin{tabular}{lc}
\hline Characteristics & Value \\
& $(n \%)$ \\
\hline Age: years (range) & $33(20-55)$ \\
Female & $160(78)$ \\
Male & $45(22)$ \\
\hline
\end{tabular}


Article History: Received: 28 October 2021 Revised: 05 December 2021 Accepted: 10 January 2022 Publication: 28 February 2022

\begin{tabular}{ll}
\hline Primary rhinoplasty & $189(92.2)$ \\
Revision rhinoplasty & $16(7.8)$ \\
Over-projected tip & $115(56)$ \\
Bulbous tip & $90(44)$ \\
\hline
\end{tabular}

TABLE II. Complications observed in patients treated with the Goldman technique

\begin{tabular}{ll}
\hline Complication & No. of patients \\
\hline Alar collapse & 1 \\
Tip under-projection & 2 \\
Bossae formation & 1 \\
Pinching of the tip & 1 \\
\hline
\end{tabular}

\section{DISCUSSION}

Surgical correction of nasal tip abnormalities is considered the greatest challenge in rhinoplasty even by experienced surgeons. It often is said that "he who masters the tip, masters rhinoplasty."

The Goldman technique, described by Goldman in his 1957 article, stated that "the importance of the mesial crura in nasal tip reconstruction" is based on the vertical division of the domes of the alar cartilages. By dividing the domes, two independent flaps are created (middle and lateral flaps), which are modified to reconstruct the shape, height, and rotation of the nasal tip.

Many modifications of this technique involving vertical division of the alar cartilages were developed later. In 1960, Lipsett suggested transecting the tip cartilages medial to the dome and excising a piece of medial crus with its overlying vestibular skin. In 1970, Safian described a technique based on transaction of the lower lateral cartilages at the level of the domes. Brennan in 1983 suggested transaction of the lateral crura lateral to the domes and overlapping of the lateral to the medial crura, with medial advancement of the lateral crura. Kridel and Konior (1991) sutured the cut ends of the alar cartilages using the external approach [12]. Foda (2001) described an over-projection technique for cases of overdeveloped alar cartilages. With his "alar setback technique," he shortened both medial and lateral crura and then reconstructed the alar cartilages to increase the stability and strength of the tip complex [13]. Other modifications of Goldman's technique were developed by Simons and coworkers, Adamson et al. [14], Constantinides et al. [15], and McLure et al. [16] in their article, Vertical Lobule Division in Rhinoplasty, reviewed the indications for each modification of vertical lobule division of the alar cartilages. 
Article History: Received: 28 October 2021 Revised: 05 December 2021 Accepted: 10 January 2022 Publication: 28 February 2022

It should be noted that the purpose of this report is not to compare the open and closed approaches to nasal tip surgery. Each approach has its distinct advantages and disadvantages, and their discussion is beyond the subject of our study [17]. The Goldman surgical technique has proved its efficacy through time. For a surgeon who favors the closed approach, this technique is, based on our study, an excellent choice for correction of both the over-projected and the broad nasal tip. We believe that the Goldman technique can have a short learning curve for a young surgeon training next to an experienced surgeon who uses the Goldman technique in his or her practice for the indications mentioned in our report.

In any case, the goal of our study, as mentioned in the report, is to show that the Goldman technique, when performed correctly, can provide a long-lasting, natural-appearing, and functionally pleasing result. Furthermore, it has a very low complication rate when performed correctly, as shown by our own low complication rate. The senior author believes that the delivery of the lower lateral cartilages provides excellent visibility for the surgical maneuvers in the nasal tip, decreasing the complication risk.

TABLE III. Mean evaluation score for the parameters studied in primary rhinoplasties

\begin{tabular}{ll}
\hline Parameter & Evaluation score \\
\hline Tip projection & 0.95 \\
Tip shape & 0.80 \\
Tip symmetry & 0.90 \\
Tip rotation & 0.85 \\
Presence and distance & 0.78 \\
of tip-defining points & \\
\hline
\end{tabular}

TABLE IV. Mean evaluation score for the parameters studied in revision rhinoplasties

\begin{tabular}{ll}
\hline Parameter & Evaluation score \\
\hline Tip projection & 0.97 \\
Tip shape & 0.88 \\
Tip symmetry & 0.94 \\
Tip rotation & 0.90 \\
Presence and distance & 0.82 \\
of tip-defining points & \\
\hline
\end{tabular}

In the literature, the bulbous (trapezoid or amorphous) tip, tip asymmetry, over-projection or under-projection, and rotation deformities of the tip are mentioned as major indications for the surgeon to use a vertical dome division technique. For the Goldman technique in particular, 
Article History: Received: 28 October 2021 Revised: 05 December 2021 Accepted: 10 January 2022 Publication: 28 February 2022

Simons and coworkers, mentioned that older patients with a ptotic tip because of skin laxity, bone resorption, or loss of connection between upper and lower lateral cartilages can largely benefit from this method. Friedman et al. found this technique very useful, especially for revision cases in which asymmetries or over-projection of the tip have occurred because of the previous surgery. They believe that with this method, tip deformities can be corrected easier than with other techniques.

From our review of the cases performed in the Functional Rhinoplasty Department of our Hospital, we see that for the majority of our patients, the surgical maneuvers in the tip are not so aggressive and that the anatomy and architecture of the alar cartilages are maintained. Interruption of their continuity (with Goldman or lateral crural overlay techniques) is observed in less than $10 \%$ of all rhinoplasty cases.

The Goldman technique is performed only after thorough estimation of the clinical indications and contraindications and if no other intraoperative efforts have provided the optimal aesthetic and functional result. The philosophy of the senior surgeon conforms to the thought of Toriumi and Tardy: "If cartilage suturing techniques prove to be inadequate, then more aggressive techniques such as dome division can be used."

It should be mentioned that Salari and Totonchi, in a randomized comparative study of 100 patients who underwent surgery with the Goldman technique and suturing of the domes, observed a statistically important difference in patient satisfaction between those treated with the Goldman technique and those who had suturing of the domes.

The described method can be used by either endonasal or open rhinoplasty. In our department, the senior surgeon always uses the closed approach, and performs the marginal and transfixion incision for delivery of the alar cartilages. We believe that with this approach, the surgeon can accurately estimate the shape, size, and resistance of the lower lateral cartilages to choose the appropriate method for the correction of the tip abnormalities.

The cephalic margins of the lower lateral crura are conservatively trimmed to promote tip refinement, with the surgeon taking care to preserve a 5- to 8-mm-wide complete strip. Overaggressive resection of the alar cartilages could compromise tip support, creating a potential for future alar pinching, alar collapse, or bossa formation.

The suturing of the two medial crura usually provides adequate support to the tip. After all, Goldman described his method as a novel technique for the improvement and stabilization of the tip without the use of grafts. However, if the surgeon observes that the newly formed central flap provides inadequate support, especially in cases of thick skin and weak cartilages, then a columellar strut is placed between the two medial crura. The strut usually is cartilaginous, harvested from the septum.

The suturing of the two medial crura with an absorbable 5-0 PDS suture is performed 2-3 $\mathrm{mm}$ under their apex to maintain the natural curvature of the nasal tip. Suturing above this level produces an unnatural, very thin or pinched tip. Our complication rate with the Goldman 
Article History: Received: 28 October 2021 Revised: 05 December 2021 Accepted: 10 January 2022 Publication: 28 February 2022

technique was low $(2.4 \%)$. All the patients with complications underwent reoperation with a successful outcome.

In the first case, which had alar collapse and external valve insufficiency due to aggressive cephalic reduction of the lateral crura, the deformity was corrected with placement of a lateral crural cartilage strut graft harvested from the auricle. We believe this complication can be generally prevented if the surgeon is very conservative in the cephalic reduction of the lateral crura. We note that the width of the lateral crura after the resection must be at least 5-8 mm, but the senior surgeon in fact very rarely leaves a lateral crural strip with a width less than $8 \mathrm{~mm}$ when performing the Goldman technique.

In the second case, which had over-projection of the tip due to excessive reduction in the height of the central flap (medial crura), an increase of tip projection was achieved with the placement of a columellar strut between the medial crura as well as the placement of a contoured auricular projection graft above the two domes.

In the third case, the bossae formation resulted from dislocation of the columellar strut (placed to support the tip), as well as from the presence of very thin skin. This deformity was corrected with resection of the bossa as well as placement of crushed cartilage to conceal contour irregularities. Thorough preoperative examination is even more important in cases of thin skin, firm alar cartilage, and interdomal bifidity because these cases involve less susceptibility to scar contracture forces responsible for bossae formation.

Our fourth complication, pinching of the tip, was experienced by a patient with very thin skin. This complication was corrected with the placement of both an onlay graft and crushed cartilage. In noses with very thin skin, crushed cartilage serves as a "camouflage" and produces a better contour. Thus, in our clinic, thin skin does not constitute a contraindication for the use of the Goldman technique. It is very important to note that to prevent pinching of the tip, the lateral crus after its height has been trimmed, must line up exactly in continuance with the edge of the new dome cartilage.

It is worth mentioning that Chang and Simons placed crushed cartilage in the infratip lobule to serve as a "cushion" between the overlying thin skin and the underlying divided domes, thereby reducing the possibility of postoperative asymmetries or bossae formation.

\section{CONCLUSION}

In our series, we had no complications such as twisted tips, tip asymmetry, or mild necrosis. Tip symmetry was pleasing, as mentioned in the Results section and the corresponding tables (Tables 3, 4). No twisted tips occurred in our series for the following reasons:

1. We insist on correct placement of the sutured medial crura. The senior surgeon gently elevates the skin of the dissected domes with a skin double hook and uses a forceps to place the medial crura complex in the midline. After the medial crura are in place, the surgeon removes the 
Article History: Received: 28 October 2021 Revised: 05 December 2021 Accepted: 10 January 2022 Publication: 28 February 2022

skin double hook and palpates the skin of the tip with his index finger to ensure that the medial crura are in the midline.

2. It is of paramount importance that the septoplasty has been performed and the septum is straight before the Goldman technique is carried out. If not, the septum itself can twist the tip.

3. Finally, the lateral crura must be of equal height to avoid twisting of the tip toward the shorter lateral crura.

Preoperative evaluation of the tip is extremely important for the surgeon to know whether one of the domes is taller. If the medial and lateral crura on one side are longer (taller dome) preoperatively, then the surgeon will have to cut more cartilage from this side, reducing the height of the lateral crura more on this side for the two lateral crura to be equal in height after the operation.

To our knowledge, our study is the first to include such a large number of patients treated with the Goldman technique (for the aforementioned indications) with such a long follow-up period. It also is worth mentioning that in our series, we used this technique to decrease tip projection, whereas most surgeons use it for its primary indication, which is to increase projection.

Evaluation of the pre- and postoperative photos using the five parameters (projection, rotation, symmetry, shape, and distance of tip-defining points) proved that the Goldman technique is effective for correction of both the over-projected and the broad nasal tip and that it produces long-lasting results in relation to the projection, rotation, and shape of the tip. In conclusion, we can safely say that according to our study results and the senior surgeon's experience, the cartilage rhinoplasty with Goldman technique still remains a very powerful tool in the surgeon's armamentarium.

\section{REFERENCES}

1. Adamson PA, McGraw-Wall BL, Morrow TA, Constantinides MS. Vertical dome division in open rhinoplasty: an update on indications, techniques, and results. Arch Otolaryngol Head Neck Surg 1994; 120:373-80.

2. Armijo BS, Brown M, Guyuron B. Defining the ideal nasolabial angle. Plast Reconstr Surg 2012; 129:759-64.

3. Behmand RA, Ghavami A, Guyron B. Nasal tip sutures part I: the evolution. Plast Reconstr Surg 2013; 112:1112-25.

4. Boccieri A. The surgical approach to theMediterranean nose. Facial Plast Surg 2010; 26:119-30.

5. Chang David CW, Simons RL. Hockey-stick vertical dome division technique for over-projected and broad nasal tips. Arch acial Plast Surg 2008;10:88-92.

6. Constantinides M, Liu ES, Miller PJ, Adamson PA. Vertical lobule division in rhinoplasty maintaining an intact strip. Arch Facial Plast Surg 2001; 3:258-63. 
Article History: Received: 28 October 2021 Revised: 05 December 2021 Accepted: 10 January 2022 Publication: 28 February 2022

7. Davis AM, Simons RL, Rhee JS. Evaluation of the Goldman tip procedure in modern-day rhinoplasty. Arch Facial Plast Surg 2004; 6:301-7.

8. Dubeta KR. Dorsal approach rhinoplasty. Int J Otorhinolaryngol Clin 2013; 5:1-23.

9. Foda H. Alar setback technique: a controlled method of nasal tip deprojection. Arch Otolaryngol Head Neck Surg 2001;127:1341-6.

10. Jeong JY. Tripod framework rebuilding in Asian nose: tip plasty using alar advancement technique, J Korean Soc Aesthetic Plast Surg 2010; 16:125-38.

11. Kim SK, Kim JC, Lee KC, Kim HS. Correction of the supratip deformity of the nose. Aesthet Surg J 2012;32:943-55.

12. Kridel RWH, Konior RJ. Controlled nasal tip rotation via the lateral crural overlay technique. Arch Otolaryngol Head Neck Surg 1991;117:411-5.

13. Leach JL, Athre RS. Four-suture-tip rhinoplasty: a powerful tool for controlling tip dynamics. Otolaryngol Head Neck Surg 2006; 135:227-33.

14. McLure TC. A modified Goldman nasal tip procedure for the drooping nasal tip. Plast Reconstr Surg 2005;87:254-60.

15. Ozturan O, Dogan R, Aksoy F, Tugrul S, Eren SB. Dorsal approach to septum in external septorhinoplasty. Ann Otol Rhinol Laryngol 2014; 26: 112-21.

16. Tardy ME, Patt BS, Walter MA. Transdomal suture refinement of the nasal tip: long-term outcomes. Facial Plast Surg 1993; 9:275-84.

17. Tebbets JB. Rethinking the logic and techniques of primary tip rhinoplasty. Otolaryngol Clin N Am 2012; 32:741-54. 